\title{
An Indirect Control Method for Sustainable Society
}

\author{
Kouji Yokoyama, Takayuki Shiose, Toshiharu Taura \\ Graduate School of Science and Technology, Kobe University \\ E-mail: 002t421n@y00.kobe-u.ac.jp
}

\begin{abstract}
Recently, we are attempting to solve environmental problems. However, the difficulty of predicting the behavior of a sustainable social system due to its very large and complexity makes it difficult to realize effective methods of solving environmental problems. In this study, a hierarchical model which is composed of a logistics layer and a decision layer is proposed, and an indirect control method of focusing on a tax is studied based on this model. The purpose of this study is to investigate the efficiency of indirect control approaches in the resources circulation system. Finally this paper discuss and conclude the effectiveness of indirect control method.
\end{abstract}

\section{Introduction}

Recently, we are faced with various environmental problems as a result of the past mass production and consumption, and are attempting to solve those problems through various efforts (e.g., restricting the amount of materials or resources consumed [1]). However, the difficulty of predicting the behavior of a sustainable social system, due to its very large scale, complexity and frequent change of its surrounding conditions, makes it difficult to realize effective methods of solving environmental problems.

Recently, as one method of analyzing such behavior, the agent-based artificial society approach has been focused on. Artificial societies are modeled by multiagent simulation method to deal with their dynamics. In fact, the artificial society approach is effective, to some extent, for analyzing the behavior of a complex society. However, from the viewpoint of a control method for realizing the desired society, conventional studies on the artificial society are insufficient. The greatest reason is that we cannot directly control each agent's mind or decision making in a real sustainable society.

In order to compensate for this defect, we introduce and extend the idea of indirect control method [2]. The indirect control method guides the system by controlling the system's boundary conditions and is a concept in opposition to the direct control method which controls the parameters in the system. By applying this method to the artificial society, we expect to realize an effective method of guiding the society to a sustainable state. Furthermore, this method can ensure each agent's freedom in decision making, which is very important in a free - economy society and may be ignored by direct control method.

The purpose of this study is to investigate the efficiency of indirect control approaches in the resources circulation system and to find a means of realizing a sustainable society.

In this study, a hierarchical model which is composed of a logistics layer and a decision layer is proposed, and an indirect control method of focusing on a tax is studied based on 
this model.

\section{Indirect control method}

Several studies have been made on the indirect control method. For example, in the study of a simulation of a prisoner's dilemma in a game, the indirect control method is used by changing the boundary conditions which are the probability of monitoring strategy in a past, compensation for a betrayed agent and sanction for a betrayer [2]. These studies reveal that the indirect control method is a good way of guiding a complex social system. However, in the resources circulation system, this method is insufficient to be applied as it is, since, a sense of agent's value changes dynamically; therefore, affecting the values indirectly can be an effective method. Hence, we must develop the indirect control method so that the evaluation function can be changed during the control. The Bi-Referential Model [3], which was previously proposed by one of the authors, is concerned with changing agent's value. Taking the idea of the model into consideration, we undertake to construct a new indirect control method. In this paper, a hierarchical model is proposed as a base for the new indirect control method.

\section{Hierarchical model}

In this paper, a hierarchical model for a resources circulation system is proposed. Figure 1 illustrates the concept. The hierarchical model consists of agents, each of which represents a consumer, a company, a municipality, and so on. In this model, the actual flow of the product or materials is described on the logistics layer. The parameters such as the price of products, the cost of processing, and the environmental load also are described on the logistics layer. In the decision layer, each agent has a sense of economy and a sense of ecology. In the decision making processes based on these dynamics, an evaluation function for decision making is used. Here, decision making means that one agent selects other agent to which the product or material is forwarded. By modeling the decision layer independent of the logistics layer, a new indirect control method for the resources circulation system can be constructed.

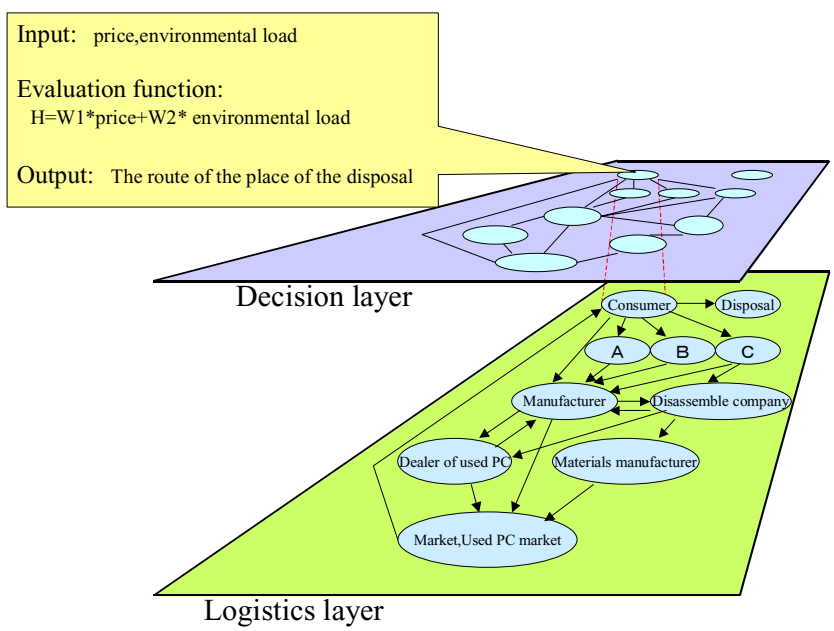

Figure 1: a hierarchical model in the resources circulatory system 


\subsection{Modeling of logistics layer}

In this level, each agent corresponds to an element of the resources circulation system, such as a consumer and a company. In this paper, we select the resources circulation system of a personal computer (PC) as an example. Figure 2 shows the concept of the PC product flow in the logistics layer.

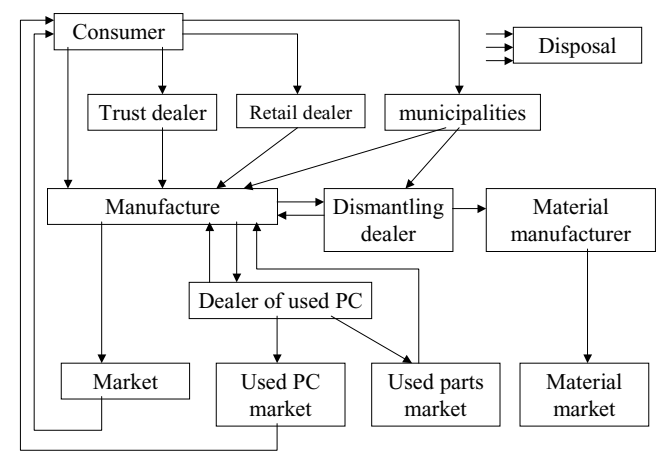

Figure 2: an example of PC flow in the logistics layer

In this paper, we refer to the consumer as a consumer agent, and to the other elements as organization agents. Each organization agent is assigned two parameters: price retrieving used PC and environmental load (Table 1). In this paper, price $\mathbf{P}$ is determined as follows:

$$
\begin{gathered}
\mathrm{P}=\mathrm{C} * \mathrm{M} \\
\mathrm{C}=\mathrm{C}_{1} /(\mathrm{a}+\mathrm{M})+\mathrm{C}_{2},
\end{gathered}
$$

where $\mathbf{C}, \mathbf{C}_{1}, \mathbf{C}_{2}, \mathbf{N}, \mathbf{M}$, and a denote total cost, fixed cost, fluctuation cost, number of products processed in a unit time, profitability, and a constant, respectively. In other words, $\mathbf{P}$ is changed dynamically by the number of products processed. The value of each parameter is an integer from 1 to 10 .

Table 1: Parameters of organization agent

\begin{tabular}{|c|l|}
\hline & Max Min \\
\hline Price & Low 10 1 High \\
\hline Environmental load & Good 10 1 Bad \\
\hline
\end{tabular}

Let $\mathbf{M}_{\mathbf{n}}$ and $\mathbf{N}_{\mathbf{n}}$ be the profitability and the number of products processed at time $\mathrm{n}$. In this paper, profitability $\mathbf{M}$ is also changed according to $\mathbf{N}$, by using

$$
M_{n}=M_{n-1}+\left(N_{n}-N_{n-1}\right) / b \text {. }
$$

b is a constant that describes the variation rate of profitability.

The environmental load indicates how much recycling and reuse are carried out by the agent. The value of this parameter is an integer from 1 to 10 . With the recycling and reuse rate defined as follows, the value of the environmental load can be selected from Table 2. The environmental load is denoted $\mathbf{E}$.

$$
r=(n-h) n
$$

Here, $\mathbf{r}, \mathbf{n}$, and $\mathbf{h}$ denote the rate of recycling and reuse, the number of products processed 
in a unit time, and the amount of waste disposed by the agent, respectively.

Table 2: Values of the environmental load

\begin{tabular}{|c|c|c|c|c|c|c|c|c|c|c|}
\hline $\mathrm{r}$ & $0.95 \sim$ & $0.9 \sim 0.95$ & $0.85 \sim 0.9$ & $0.8 \sim 0.85$ & $0.7 \sim 0.8$ & $0.6 \sim 0.7$ & $0.45 \sim 0.6$ & $0.3 \sim 0.45$ & $0.15 \sim 0.3$ & $\sim 0.15$ \\
\hline $\mathrm{E}$ & 10 & 9 & 8 & 7 & 6 & 5 & 4 & 3 & 2 & 1 \\
\hline
\end{tabular}

\subsection{Modeling of decision layer}

Each agent is assumed to make a decision based on its own values represented on two sense axes: a sense of economy and a sense of ecology. The former is denoted W1 and the later $\mathbf{W} \mathbf{2}$. Table 3 gives the values of these parameters.

Table 3: Sense of value

\begin{tabular}{|c|c|}
\hline & Max $\sim$ Min \\
\hline W1 & High $10 \sim 1$ Low \\
\hline W2 & High $10 \sim 1$ Low \\
\hline
\end{tabular}

In this study, a consumer agent selects an other agent with the largest value of the evaluation function $\mathbf{H}$.

$$
\mathrm{H}=\mathrm{W} 1 * \mathrm{P}+\mathrm{W} 2 * \mathrm{E}
$$

\section{Simulation}

In this study, we simulated the resources circulation system for the recycling of PCs and investigated the conventional methods of indirectly controlling the system. The established simulation software, called ARENA, is adopted to represent the resources circulation system. Here, we deal with taxation of organization agents as an example of indirectly controlling the system.

\subsection{Setup of simulation}

In this study, we assume that a tax is aimed at increasing the cost of taking back used PCs. When an agent is imposed a tax, the price is determined using

$$
\mathrm{P}=\mathrm{C} * \mathrm{~T} * \mathrm{M}
$$

where $\mathbf{T}$ is the tax rate.

In the simulation, we investigate the effectiveness of imposing a tax, depending on the number of imposed agents and the time interval to impose a tax. For the former, we conduct two kinds of experiments: collection of the tax from all organization agents, collection of the tax from organization agents whose rate of recycling and reuse is less than a certain criterion (the value is 0.5 in this experiment). For the latter, we adopt three kinds of experiments: every 20 times, every 40 times, and every 60 times. In total, there are six experimental patterns.

\subsection{Result and considerations in this simulation}

The results do not indicate that indirect control decreases the amount of disposal of PCs. However, the number of PCs that consumer agents decide to dispose shows some change. Figure 3 shows the simulation results. The vertical axis represents the standard deviation of 
the disposal rate of consumer agents. The disposal rate is determined as follows:

$$
\mathrm{s}=\mathrm{h} / \mathrm{n} \text {, }
$$

where $\mathbf{s}, \mathbf{h}$ and $\mathbf{n}$ are the scrap rate, the amount of waste disposed by consumer agents in a unit time, and the total number of used PCs in a unit time.

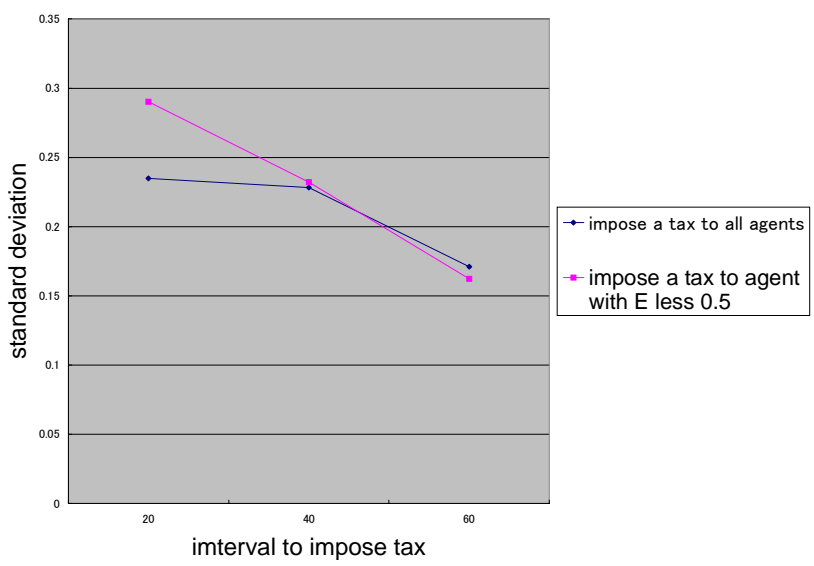

Figure 3: Simulation result of consumer agent's action

Figure 3 shows that the standard deviation decreases as the time interval increases. In other words, when the time interval to impose a tax is long, the number of used PCs disposed by consumers is more stable. The reason is that imposing a tax affected the agent's sense of economy. It is advantageous for a company which recycles used PCs to supply used PC more stable. From this point of view, the imposition of a tax is an effective one of indirect control method.

\section{Conclusion and future prospects}

In this paper, we explored the problem of the resources circulation system. We explain an indirect control to realize an efficient resource circulation system. We proposed a hierarchical model in order to deal with the sense of values of agents. Furthermore, we developed such a model and analyzed the simulation result of the efficiency of the indirect control of imposing a tax. The possibility of indirect control was demonstrated.

In this experiment, the evaluation function of the agent was not changed during the control process. We are currently trying to extend the system so that the evaluation function is changed according to the indirect control information.

\section{References}

[1]Kohmei Halada. Progress of Ecomaterials Research towards Sustainable Society. EcoDesign'99, pp.212 215, 1999

[2]Shiose,Sawaragi,Katai,Okada. Dynamics of Receprocal Learning by Bi-Referential Model within Multiagent Systems. Proceedings of the 1998 IEEE International Comference on Systems, Man, and Cybernrtics, Vol.4, pp.4045 4050. 1998

[3]Hiroshi Deguchi, Agent based Approach for Social Complex Systems, Community Computing and Support Systems, LNCS 1519, Springer, pp.62 77, 1998 